\title{
CANCELAMENTOS DE CIRURGIAS EM UM HOSPITAL DA REGIÃO METROPOLITANA DO RECIFE, 2017: A IMPORTÂNCIA PARA A CONTABILIDADE DE CUSTOS
}

\author{
M.M.M.CARVALHO ${ }^{1}$ e U.C.T. LAGIOIA ${ }^{2}$ \\ ${ }^{1}$ Universidade Federal de Pernambuco, Centro de Ciências Sociais Aplicadas, Departamento \\ de Economia, Mestranda no Programa de Pós-Graduação em Gestão e Economia da Saúde \\ (PPGGES) e Enfermeira Gerente do Bloco Cirúrgico do Hospital Miguel Arraes \\ ${ }^{2}$ Universidade Federal de Pernambuco, Centro de Ciências Sociais Aplicadas, Departamento \\ de Economia, Docente no Programa de Pós-Graduação em Gestão e Economia da Saúde \\ (PPGGES)
}

E-mail para contato: monicamagalhaesc@gmail.com

\begin{abstract}
RESUMO - No âmbito da saúde, a Contabilidade de Custos se faz de suma importância frente à necessidade de alocação dos recursos escassos e demanda crescente. Este estudo tem como lócus de pesquisa um hospital público da Região Metropolitana do Recife, com ênfase nas suspensões das cirurgias clínicas. A suspensão de cirurgia acarreta prejuízos ao paciente, e também gera prejuízos financeiros para os hospitais. Objetiva-se nesta pesquisa identificar o quantitativo de cirurgias canceladas e descrever quais os motivos que levaram aos cancelamentos das cirurgias. Os dados foram coletados utilizando-se planilha de cancelamentos de cirurgias da instituição e formulário elaborado pelas autoras, e analisados quantitativamente. O periodo estudado foi de 05 meses (junho a outubro de 2017). Foram suspensas 352 cirurgias (11,48\% das cirurgias programadas). Os meses com o maior número foram outubro (125), agosto (62) e julho (61). Os motivos mais registrados foram: tempo cirúrgico excedido (42), cirurgia de emergência (36), ocupação de sala (25) e mudança de conduta (21). Como principais resultados, observou-se que parte desses cancelamentos eram evitáveis. Os pacientes que tiveram suas cirurgias suspensas permanecerem no hospital, ocasionando um aumento dos custos de internação. Diante do exposto, faz-se necessário um melhor planejamento e avaliação clínica prévia para a melhoria do desempenho da organização, visando à diminuição de gastos frente aos cancelamentos de cirurgia.
\end{abstract}

Palavras chaves: gestão de custos, unidade hospitalar, suspensão de cirurgia

ABSTRACT-In health, Cost Accounting is extremely important in the face of the need to allocate scarce resources and increasing demand, it has the task of managing accurate and quick information for the administration. This study has as a locus of research a public hospital of the Metropolitan Region of Recife, with emphasis on surgical clinics. 
The suspension of surgery causes harm to the patient, and also generates financial losses for the hospitals. This study aims to identify the quantity of surgeries canceled and describe what the reasons which led to the cancellation of surgeries. The data were collected using a spreadsheet of cancellations of surgeries of the institution and form drawn by the authors, and analyzed quantitatively. The studied period was 05 months (June to October 2017). There were suspended 352 surgeries (11,48\% of surgeries scheduled). The months with the largest number were October (125), August (62) and July (61). The reasons more recorded were: surgical time exceeded (42), emergency surgery (36), room occupancy (25) and a change of conduct (21). It should be noted that part of these cancellations were avoidable. The patients who had their surgeries suspended and remain in the hospital, should increase the costs of hospitalization. It is necessary to have a better planning and clinical evaluation prior to the improvement of the organization performance, aiming at the reduction of expenses ahead to cancellations of surgery.

Key words: Management of costs, hospital unit, suspension of surgery

\section{INTRODUÇÃO}

A Contabilidade de Custos teve como objetivo inicial buscar soluções para os problemas de mensuração monetária dos estoques e do resultado (CREPALDI, 2009 e MARTINS, 2010). Para Martins (2010, p. 22) a Contabilidade de Custos tem duas funções relevantes: "o auxílio ao Controle e a ajuda às tomadas de decisões. " O autor ainda complementa que: " o conhecimento dos custos é vital para saber se, dado o preço, o produto é rentável ou não e se é possível reduzi-los (custos). " A despeito da gestão de custos, Sivieiro (2010) destaca a importância desta ferramenta para a tomada de decisões nas empresas, pois uma boa gestão de custos propicia uma melhor visualização dos gastos, identificando as atividades envolvidas na prestação do serviço, exercendo uma ação eficaz de controle de custos. No entanto, a contabilidade de custos não está restrita apenas as empresas comerciais e industriais. E, no âmbito da saúde, se faz de suma importância frente à necessidade de alocação dos recursos escassos e demanda crescente. Rutledge (1996) apud Lagioia et all (2008) afirma que o meio ambiente hospitalar vem progressivamente demandando um maior entrosamento entre os médicos e os administradores dessas instituições no que diz respeito ao controle dos custos e à melhoria da qualidade. Neste sentido, Beulke e Bertó (1997) destacam a relevância do controle de custos para a sobrevivência de hospitais, que dependem de forma acentuada das receitas geradas através do Sistema Único de Saúde (SUS).

Quanto à contabilidade de custos na área da saúde, Falk (2001) afirma que esta facilita um controle mais preciso dos custos, com a finalidade de diminuição ou substituição de itens que se façam mais onerosos na compostura dos custos. Permitindo assim, entre outras coisas, melhor análise de lucros, planejamento e gerenciamento de pessoal (médico e não médico) em termos de produtividade e perfil operacional de atendimento. Este estudo tem como lócus de pesquisa o Hospital Miguel Arraes (HMA), no município do Paulista - Pernambuco. O referido hospital, tem se mostrado uma instituição de suma importância para o atendimento de pacientes vítimas de traumatismo na região, sendo realizadas 530 cirurgias por mês, e gerando benefício a mais de 1 milhão de pessoas que residem na área norte da Região Metropolitana e Zona da Mata Norte (PERNAMBUCO, 2016). "A unidade conta com 180 leitos, sendo 29 deles de UTI, e oferece serviços de emergência 24 horas em traumatoortopedia, clínica médica e cirurgia geral" (PERNAMBUCO, 2016). Segundo Cámio et al. 
(1995) apud Antonio, Munari e Costa (2002): o ato cirúrgico consiste, para o paciente, um dos momentos mais críticos no processo terapêutico, tendo em vista o medo do desconhecido e da anestesia, a sua complexidade e o próprio risco inerente a qualquer procedimento desta natureza CÁMIO et al. (1995, p. 40) apud ANTONIO, MUNARI, COSTA, (2002, p.34). Estudos a respeito do ato cirúrgico trazem que tal procedimento causa alterações na rotina de vida de uma pessoa, consequente afastamento das atividades laborais, do lar e necessidade de mobilização de recursos financeiros, por conseguinte, o seu cancelamento gera frustração.

Pacientes diante desta situação demonstraram sentimento de revolta, desconfiança na equipe, insegurança e intenso estresse; sentimentos negativos e de conformismo; preocupação e falta de controle da situação (PERROCA, JERICÓ e FACUNDIN, 2007). O cancelamento de um procedimento possivelmente não gera só transtornos ao paciente, mas também à instituição hospitalar, que deve ter um planejamento interrompido, ocasionando muitas vezes o prolongamento da estadia do paciente no hospital e com isso todos os custos advindos deste. Diante do exposto, e sabendo da necessidade crescente que as instituições de saúde pública tem de manter o controle de custos, esta pesquisa objetivou identificar o quantitativo de cirurgias canceladas e descrever quais os motivos que levaram aos cancelamentos das cirurgias.

\section{MATERIAIS E MÉTODOS}

Estudo do tipo observacional, retrospectivo com abordagem quantitativa. Foram verificados os cancelamentos de cirurgia de 05 meses do ano de 2017. A presente pesquisa possui natureza exploratória, com análise de dados qualitativa. O estudo foi desenvolvido no Hospital Miguel Arraes, Paulista-PE. O período de coleta de dados foi entre os meses de junho a outubro de 2017. Neste interstício, buscou-se informações nas planilhas de cancelamento de cirurgias disponíveis no Centro Cirúrgico do hospital (obtendo assim a justificativa expressa nesse documento) e também no censo de cirurgias realizadas. Os dados foram tabulados em formulário elaborado pelas pesquisadoras. A população foi composta por todos os pacientes que tiveram as cirurgias programadas no período analisado. A amostra foi formada pelos pacientes que as cirurgias foram suspensas, durante o período citado. Foram avaliados o quantitativo de cirurgias canceladas, bem como os motivos que levaram aos cancelamentos das mesmas.

\section{RESULTADOS E DISCUSSÃO}

A partir dos motivos de cancelamento, foram classificados todos os dados e somados um a um mostrando um total mensal durante o período total da coleta. Das 3067 cirurgias programadas, 352 foram suspensas (11,48\%). O gráfico 1 mostra o número de cancelamentos distribuídos por meses. Os meses com o maior número de cancelamento foram outubro (125), agosto (62) e julho (61). No gráfico 2, pode-se identificar uma série de motivos que foram descritos na planilha de suspensão de cirurgia. Dentre estes, os mais registrados foram: tempo cirúrgico excedido (42), cirurgia de emergência (36), ocupação de sala (25) e mudança de conduta (21), condições clínicas desfavoráveis (12), pré-operatório inadequado (6), demanda da urgência (6), outros (6) e falta de jejum (5).

Entende-se o uso de tempo cirúrgico excedido quando a cirurgia anterior teve um tempo cirúrgico maior do que o programado, impossibilitando assim o início de outra cirurgia. O motivo cirurgia de emergência é usado quando a cirurgia tem de ser cancelada porque uma emergência (prioridade) teve que ser admitida no Centro Cirúrgico. O motivo ocupação de 
sala poderia ser distribuído também em cirurgias de emergência e tempo cirúrgico excedido, devido estas muitas vezes serem o motivo da ocupação de sala. A mudança de conduta também poderá está ligada ao pré-operatório inadequado, e poderia ser evitado em muitos casos, Falta de jejum do paciente poderá indicar o não preparo do paciente por parte da equipe de saúde, ou mesmo o desconhecimento do paciente em estar em programação cirúrgica (caracterizando falha de comunicação).

Gráfico 1 - Cancelamento de Cirurgia - Junho a Outubro/2017 - Hospital Miguel Arraes.
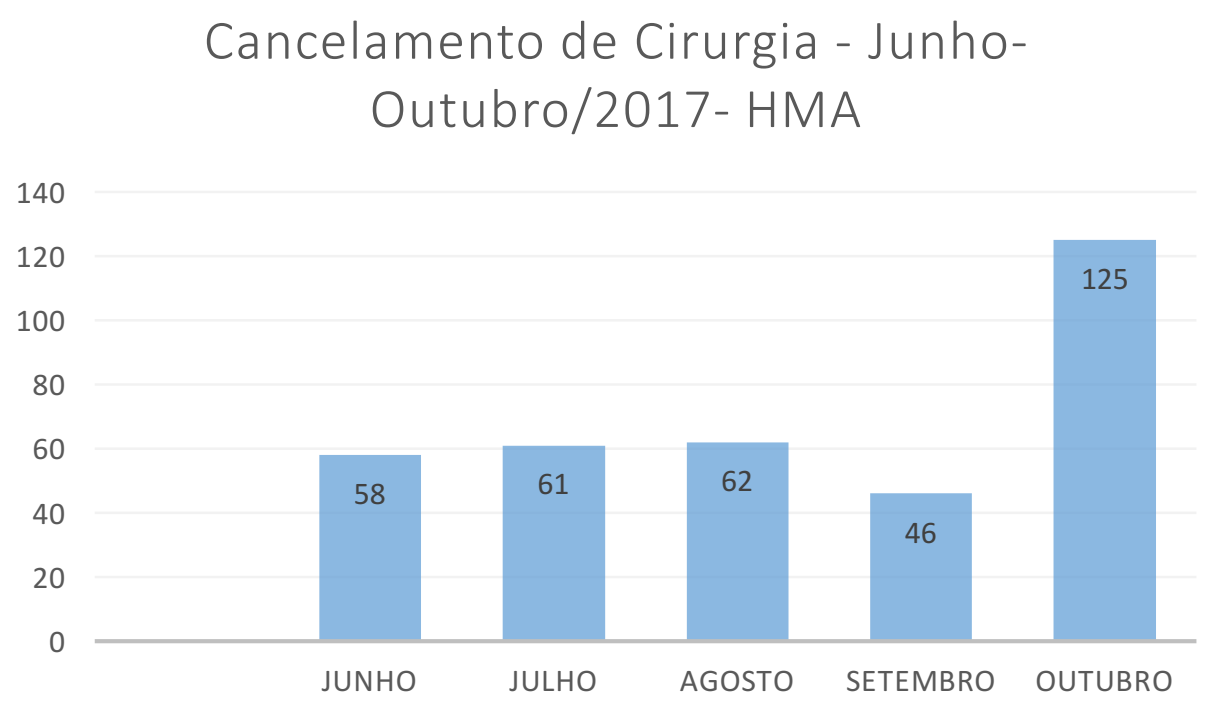

Fonte: Planilha de Cancelamentos de Cirurgia - HMA

Gráfico 2 - Motivos dos Cancelamentos de Cirurgia- Junho a Outubro/2017 - Hospital Miguel Arraes.

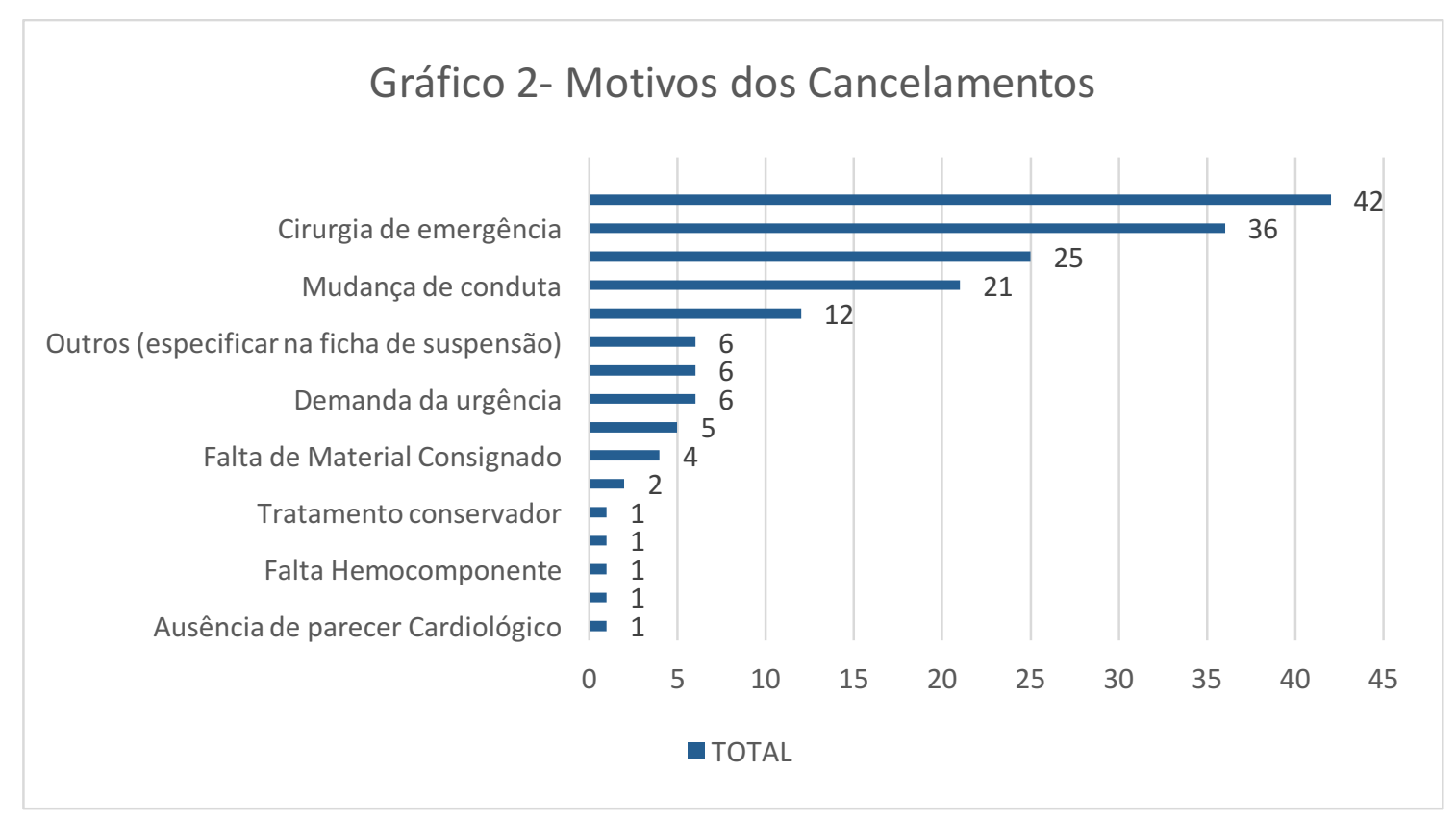

Fonte: Planilha de Cancelamentos de Cirurgia - HMA 


\section{CONCLUSÃO}

Faz-se necessário maiores investigações nessa área a fim de se conhecer as causas que determinaram o cancelamento dessas cirurgias. Considerando se tratar de um hospital de urgência, a maioria dos pacientes já estão internados, portanto, a suspensão da cirurgia poderá levar ao aumento de tempo de permanência no hospital. Assim, os custos devem aumentar em proporção aos dias internados. A pesquisa espera gerar conhecimento a respeito dos principais motivos que levam aos cancelamentos de cirurgias. Com esses dados busca-se gerar ferramentas para melhorar a gestão dos blocos cirúrgicos trazendo benefícios não somente para o hospital, como também para os pacientes.

\section{REFERÊNCIAS}

ANTONIO, Priscila da Silva; MUNARI, Denize Bouttelet; COSTA, Hérica Kelly. FATORES GERADORES DE SENTIMENTOS DO PACIENTE INTERNADO FRENTE AO CANCELAMENTO DE CIRURGIAS. Revista Eletrônica de Enfermagem, Goiânia, v. 4, n. 1, dez. 2002. ISSN 1518-1944. Disponível em: <https://www.revistas.ufg.br/fen/article/view/744/806 $>$. Acesso em: 04 jan 2017.

BEULKE, Rolando; BERTÓ, Dalvio José. Gestão de custos e resultado na saúde: hospitais, clínicas, laboratórios e congêneres. São Paulo: Saraiva, 1997.

CREPALDI, Silvio Aparecido. Curso básico de contabilidade de custos. 4.ed. - São Paulo: Atlas, 2009.

FALK, James Anthony. Gestão de Custos para Hospitais: Conceitos, Metodologias e Aplicações. São Paulo: Atlas, 2001.

LAGIOIA, Umbelina Cravo Teixeira et al. A gestão por processos gera melhoria de qualidade e redução de custos: o caso da unidade de ortopedia e traumatologia do hospital das clínicas da Universidade Federal de Pernambuco. Rev. contab. finanç. [online]. 2008, vol.19, n.48, pp.77-90. ISSN 1808-057X. Disponível em: < http://dx.doi.org/10.1590/S1519-70772008000300007>. Acesso em: 10 nov. 2016.

MARTINS, Eliseu. Contabilidade de Custos. 10. ed. São Paulo: Atlas, 2010.

PERNAMBUCO. SECRETARIA DE SAÚDE DO ESTADO DE PERNAMBUCO. (Org.). Hospital Metropolitano Norte - Miguel Arraes de Alencar. Disponível em: $<$ http://portal.saude.pe.gov.br/unidades-de-saude-e-servicos/secretaria-executiva-deatencao-saude/hospital-metropolitano-norte>. Acesso em: 20 dez. 2016.

PERROCA, Márcia Galan; JERICO, Marli de Carvalho e FACUNDIN, Solange Diná. Monitorando o cancelamento de procedimentos cirúrgicos: indicador de desempenho organizacional. Rev. esc. enferm. USP [online]. 2007, vol.41, n.1, pp.113-119. ISSN 0080-6234. Disponível em: < http://dx.doi.org/10.1590/S008062342007000100015>. Acesso em: 22 out. 2016. 


\section{$5^{\circ} \mathrm{EBIT}$}

\section{ENCONTRO BRASILEIRO PARA}

SIVIERO, André Luiz Palú. A IMPORTÂNCIA DA GESTÃO DE CUSTOS COMO INSTRUMENTO DE COMPETITIVIDADE EM INSTITUIÇÕES DE ENSINO SUPERIOR: UM ESTUDO DE CASO NO CENTRO UNIVERSITÁRIO EURÍPIDES DE MARÍLIA - UNIVEM. REGRAD - Revista Eletrônica de Graduação do

UNIVEM - ISSN 1984-7866, [S.1.], v. 3, n. 1, dec. 2010. ISSN 1984-7866. Disponível em: $<$ http://revista.univem.edu.br/REGRAD/article/view/244> $>$. Acesso em: 25 out 2016. 